\title{
Use of Panel Code Modeling in a Framework for Aircraft Concept Optimization
}

\author{
Amadori, K. ${ }^{*}$ and Jouannet, C. ${ }^{\dagger}$ \\ Linköping University, Linköping, 581 83, Sweden \\ Krus, $\mathrm{P}$. \\ Linköping University, Linköping, 581 83, Sweden
}

In this study the use of a high-order panel code within a framework for aircraft concept design is discussed. The framework is intended to be a multidisciplinary optimization tool to be adopted from the very beginning of the conceptual design phase in order to define and refine the aircraft design, with respect to its aerodynamic, stability and control, structure and basic aircraft systems. The presented work is aimed at developing a module for aerodynamic analysis of concepts as a basis for a direct search optimization of the concept layout. The design criterion, used in the example presented here, is to minimize the maximum take-off weight required to fulfil the mission. Classic and simple equations are used together with the data generated by the panel code solver to calculate the aircraft's performances. Weights are calculated by means of statistical group weight equations, but the weight could also be calculated from a CAD-model. The design of an Unmanned Combat Air Vehicle is used as test case for three different optimization algorithms: one gradient method based (Fmincon), one non-gradient based (Complex) and one Genetic Algorithm (GA). Comparison of results and performances shows that the Genetic Algorithm is best fitted for the specific problem, having the by far best hit rate, even if it is at a cost of longer computing time. The Complex algorithm requires less iterations and is also able to find the optimum solution, but with a worse hit rate, while Fmincon can not reach to a global optimum. The suggested optimized configuration for the aircraft is very similar to the Boeing $\mathrm{X}-45 \mathrm{C}$ and Northrop Grumman X-47B.

\section{Nomenclature}

$=$ angle of attack

$=$ semi wing span

$=$ specific fuel consumption

$=$ parasite drag coefficient

$=$ induced drag coefficient

$=$ skin friction coefficient

$=$ lift coefficient

$=$ lift coefficient as function of the angle of attack

$=$ lift coefficient at zero angle of attack

$=$ moment coefficient

$=$ moment coefficient as function of the angle of attack

$=$ root chord length

$=$ tip chord length

$=$ aerodynamic drag force

= Osvald's Efficiency Factor

$=$ endurance

\footnotetext{
*PhD Student, Department of Mechanical Engineering, kriam@ikp.liu.se,

${ }^{\dagger}$ Ph.D., Department of Mechanical Engineering, chrjo@ikp.liu.se, AIAA Member.

${ }^{\ddagger}$ Professor, Department of Mechanical Engineering, petkr@ikp.liu.se.
} 


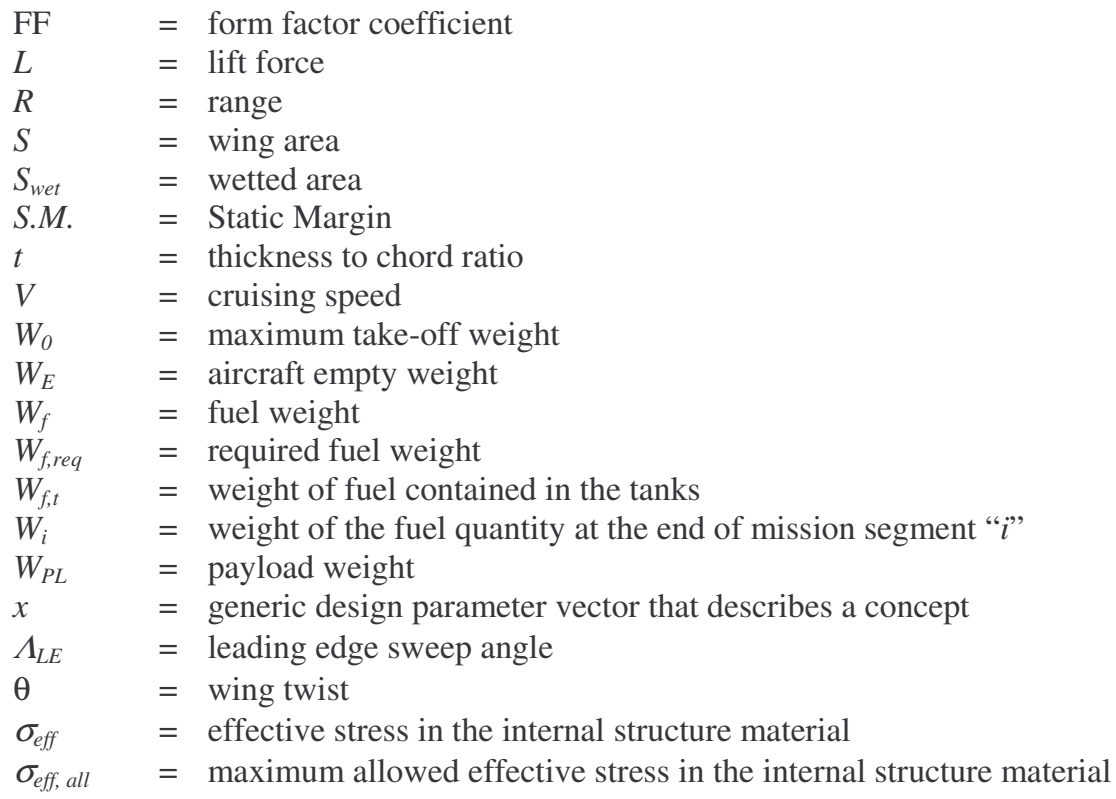

\section{Introduction}

A

IRCRAFT manufacturers today face a variety of challenges. The most important being the stiffening competition that press down the price tag for each sold aircraft and reduces the profit margin ${ }^{1}$, and the risk that new subjects located in low-labour-cost countries rise on the market and offer competitive products at extremely low prices $^{2}$. This means that there is a need to reduce the time-to-market for every new aircraft developed. To be able to do so, new and more efficient approaches to the design process are to be introduced. This is one of the reasons why design optimization has seen a growing interest in the recent years. In particular true Multi-Disciplinary Optimization (MDO) has a great potential. Moreover, constantly cheaper and more powerful computers, and the increasing computational capacity is greatly welcome for MDO applications. ${ }^{3}$

In particular the conceptual design phase could take advantage of a novel methodology, that would not be based on empirical or semi-empirical equations to estimate e.g. weights, performances, costs, and loads, but relay on analytical models to a greater extent. There are many examples of how MDO can be successfully adopted in the early development phases of new aircrafts. ${ }^{1-6}$ A field where it is felt that much work can still be done is in the flexibility of the optimization tools, in order not to have a tool that is tailor made for one specific type of aircraft only, but can be adopted on a range of types as wide as possible.

With all this in mind, a novel design framework is being developed at Linköping University that aims at being flexible enough to permit the study of a variety of different types of airplanes and that includes modules that allows to optimize the design of all main sub-systems.

\section{Design Framework}

The design framework being developed ${ }^{7}$ is intended to be a tool that permits the optimization of the design and layout of concepts during the conceptual design phase. As illustrated in Fig. 1 it comprises many modules that cover most aircraft's sub-functions and each one of them can be run independently or together with others. The different modules can range from being more elaborated, as the panel code used for the aerodynamics calculations, down to very simple handbook formulas. It is possible to develop a module alone and test it in the framework using very simple models for the other modules. The other modules will then work as support only, to close the design space. In this way the number of parameters to be optimized is greatly reduced as well as the time required to complete the optimization. For instance, as it will be shown in the presented study, running the aerodynamic calculations alone requires that the aircraft weight is calculated in order to complete the performance prediction. Not using the CAD modeling tool, the weight is estimated by means of approximate statistical group weight method ${ }^{8}$. The performance prediction can be carried out through simple sizing methods as the one described in Ref. 8 or by means of complete mission simulations. Once one module is ready and positively tested, it can be added to the framework and used together with all the others. 


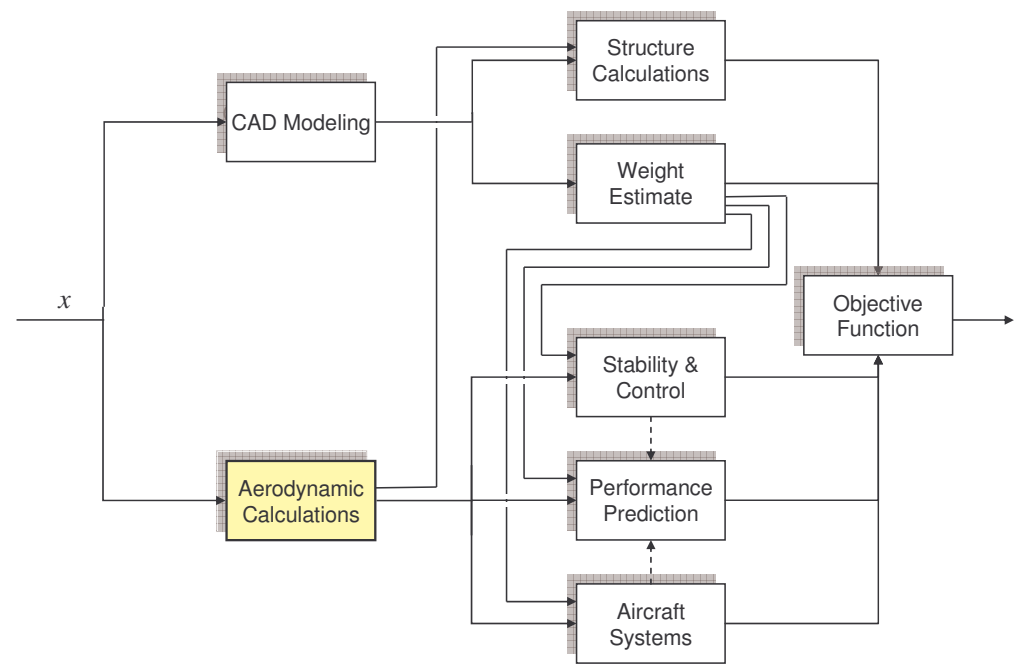

Figure 1. The design framework

\section{Aerodynamic Calculations Module Structure}

The presented aerodynamic calculation module will be used within the previously discussed framework to analyze the aerodynamics of aircraft concepts. The module has not been integrated in the framework yet; therefore some parameters need to be calculated in more simple ways, as it will be described in the following paragraphs.

The tool uses different computer applications that are linked together. In the following paragraphs it is illustrated how each application is used.

\section{A. Aerodynamic Module Logical Layout}

The aerodynamic module is governed by MatLab algorithms. Figure 2 shows how the different functions are linked together and how they interact. The main function Optindata.m is responsible for running the optimisation and is the function that decides which set of parameters are to be analyzed and that are sent to the function Opt_Panair.m, which is the one that acts as an interface between MatLab and PANAIR. The function Input_Panair.m creates the input text file with the proper formatting ${ }^{9,10}$ that PANAIR requires to run an analysis of the present aircraft configuration. Once the analysis is completed and the result file is ready, Opt_Panair.m scans it and retrieves the data needed for calculating the objective function value that is finally returned to function Optindata.m. Opt_Panair.m is also the function where the weight estimation takes place. Using an approximate group weight method as described in Raymer ${ }^{8}$, the wing weight and the fuselage weight are calculated. The payload weight is constant as well as the weight of control and mission system hardware.

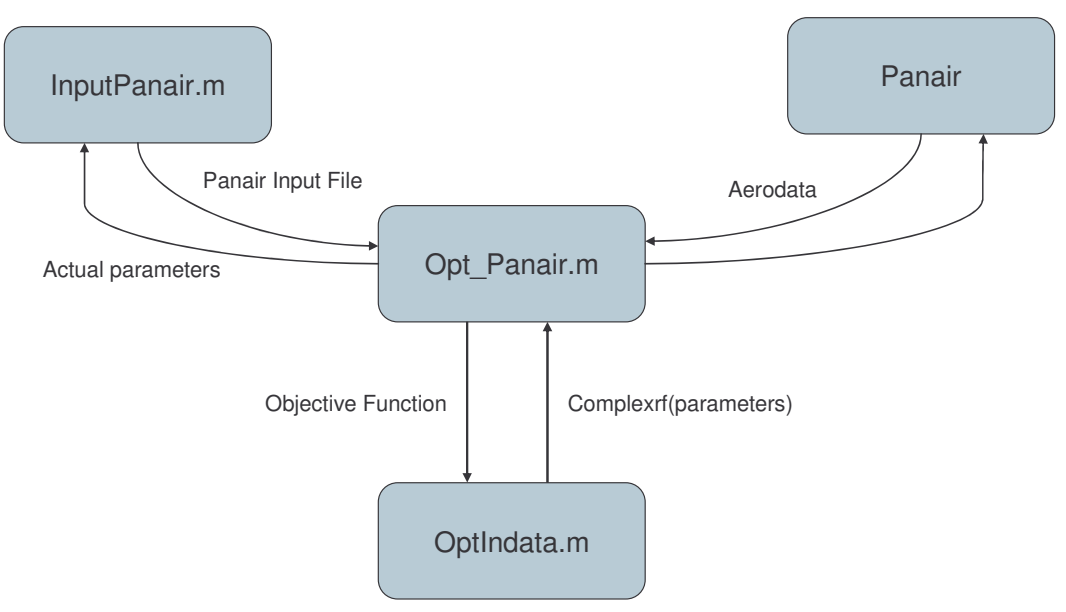

Figure 2. Logical links of MatLab functions

\section{B. PAN AIR}

According to Erikson L. "panel methods are numerical schemes for solving (the Prandtl-Glauert equation) for linear, inviscid, irrotational flow about aircraft flying at subsonic or supersonic speeds". They are not as precise as 
modern CFDs can be, but they have other advantages, especially for applications like the one here presented. The main issue is that, at present time, CFDs still require much longer computing time and are hardly feasible for robust iterative applications. Moreover, considering that during a conceptual design phase the aircraft geometry and its outer shape is not precisely defined and that the detail level is quite rough, it is clear that it can be unnecessary to adopt tools that have a much higher accuracy. Moreover CFDs requires the space around the studied body to be accurately meshed, while for a panel code it is sufficient to approximate the aircraft outer surfaces with proper rectangular panels. It should be kept in mind that in this framework PANAIR is used mainly to compare the effectiveness of different concepts with each other, rather than to gather exact and absolute figures of their aerodynamic efficiency. If much powerful and faster computers were available or if higher accuracy was required, PANAIR could be substituted with other solvers, thanks to the modular nature of the framework.

The shape of the aircraft is parametrically described in the same way as presented by Jouannet et al. ${ }^{11}$. Figure 3 shows an example of an input model for PANAIR of a generic concept with its surfaces approximated with appropriate panels.

Since PANAIR is able to analyze the same aircraft for different angles of attack, each concept is studied at two angles of attack, in order to determine the slope of the lift, drag and pitching moment coefficient curve as function of $\alpha$. The output file produced by PANAIR contains a great amount of data among which only a small part needs to be retrieved. Namely they are:

- total lift coefficient, $C_{L}$, for each $\alpha$-value considered;

- total induced drag coefficient, $C_{d i}$, for each $\alpha$-value considered;

- total pitching moment coefficient, $C_{m}$, for each $\alpha$-value considered.

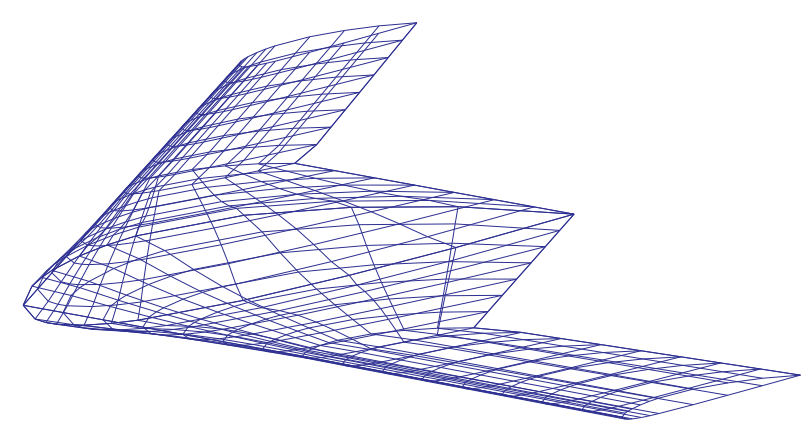

Figure 3. PANAIR input model example

In the following paragraph it will be discussed how these data are used to compute the objective function.

\section{Weight and Performance Estimation}

In order to calculate the weight required for the aircraft to be able to complete the mission, the Breguet equation ${ }^{8}$ has been used:

$$
R=\frac{V}{C} \frac{L}{D} \ln \frac{W_{i-1}}{W_{i}}
$$

or in case of loiter:

$$
E=\frac{1}{C} \frac{L}{D} \ln \frac{W_{i-1}}{W_{i}}
$$

The lift-to-drag ratio during cruise is calculated as following:

$$
\frac{L}{D}=\frac{C_{L} q S}{\left(C_{d i}+C_{d 0}\right) q S}=\frac{c_{L, \alpha} \cdot \alpha+c_{L, \alpha=0}}{C_{d i}+C_{d 0}}
$$

where $C_{L}$ is directly obtained from PANAIR. The parasite drag coefficient $C_{d 0}$ is calculated as following: 


$$
C_{d 0}=c_{f}(F F) \frac{S_{w e t}}{S}
$$

where $c_{f}$ is a skin friction coefficient, which value can be found in tables as the ones reported in Ref. 8 and Ref. 12. $F F$ is a form factor that depends on the shape of the aircraft ${ }^{8}$.

Osvald's Efficiency factor $e$ is required to be able to calculate the induced drag coefficient. $e$ is given by:

$$
e=\frac{\left(c_{L, \alpha} \cdot \alpha+c_{L, \alpha=0}\right)^{2}}{\pi \cdot C_{d i} \cdot A R}
$$

where $A R$ is the aspect ratio. Please note that the parameter $C_{d i}$ in equation (5) is the induced drag value specific for one angle of attack. Since PANAIR allows studying the same geometry at different angles of attack simultaneously, $e$ can be calculated for each one of the $\alpha$-values considered and then calculate a mean value. The induced drag coefficient for the considered cruise condition is then obtained from the following equation:

$$
C_{d i}=\frac{\left(c_{L, \alpha} \cdot \alpha+c_{L, \alpha=0}\right)^{2}}{\pi \cdot e \cdot A R}
$$

Accordingly to Ref.8, engine dimensions and parameters have been scaled up or down using as scale factor the ratio between the required thrust and the actual thrust of the reference engine. For the present case there is a list of nine different engines to choose from. Given the required thrust, the engine that fits best is selected and thereafter geometrical dimensions, weight and specific fuel consumption are set.

An important constraint to the optimization is given by the limit stress requirements in the structure. Since the structure has to be resistant enough to withstand all the flying loads, this requirement avoids that the optimizer gives a solution where all thicknesses are at the minimum allowed value in order to reduce drag as much as possible. Since the CAD module that incorporates the finite element analysis was not used here, the structure calculation is merged with the weight estimation of wing and fuselage. The equations used for estimating the empty weights of wing and fuselage are obtained from statistical analysis of existing aircrafts ${ }^{8}$, so that the weight calculated comprises by itself the need for enough structural strength.

$W_{0}$ can be considered being the sum of the required fuel weight $\left(W_{f}\right)$ that is calculated from the mission profile as a product of fractions of $W_{0}^{8}$, the aircraft's empty weight $\left(W_{e}\right)$, the payload weight $\left(W_{p}\right)$, the engine weight $\left(W_{\text {eng }}\right)$ and the control system weight $\left(W_{C T R L}\right)$, as described by the following equation:

$$
W_{0}=W_{e}+W_{f}+W_{p}+W_{\text {eng }}+W_{\text {CTRL }}
$$

The framework described in Section II will comprise a module for control system design and optimization. So far it has not been included and therefore the weight of actuators, pipelines, reservoirs, tanks and other components has been included into the aircraft empty weight, while the computer hardware weight is the one labelled $W_{C T R L}$, and it has been estimated by comparison with known flying aircrafts.

\section{Optimizer}

The data gathered from PANAIR are finally merged into an objective function (ObjFun) that expresses quantitatively the goodness of one given concept. The objective function takes into account both take-off weight of the aircraft and how close some of its predicted characteristics are to predefined wanted values, as in equation (7):

$$
\text { ObjFun }=K_{1}\left(\frac{W_{0}+P_{T C}+P_{\alpha}+P_{C_{m}}+P_{\text {fuel }}+P_{S}}{W_{0, \text { nom }}}\right)^{\beta_{1}}+K_{2}\left(\frac{B}{B_{\text {nom }}}\right)^{\beta_{2}}
$$

The factors $K_{1}, K_{2}, \beta_{1}$ and $\beta_{2}$ are constants used to assign the wanted weight to the two terms of the equation. $W_{0, \text { nom }}$ and $B_{\text {nom }}$ are the take-off weight and the span width of a nominal configuration used as reference. $P_{T C}, P_{\alpha}$, 
$P_{C m}, P_{f u e l}$, and $P_{S}$ are penalties that are used, together with constraints, to lead the optimizer away from unwanted configurations. The definition of the related penalty functions can be found in the following Section III-E. In the considered mission there are only straight-line cruises and there is no account for manoeuvring performances. Thus the aim of the second term of the objective function is to award configurations with better rolling rate that is, in first approximation, inversely proportional to the span width.

The optimization problem can be described as following:

$$
\begin{aligned}
& \min \text { ObjFun } \\
& \text { s.t. } \\
& x_{\min } \leq x \leq x_{\max } \\
& R \geq R_{\text {req. }} \\
& E \geq E_{\text {req. }} \\
& \sigma_{\text {eff }} \leq \sigma_{\text {eff, all. }} \\
& c_{m_{\alpha}}<0 \\
& \alpha_{\min }<\alpha_{\text {cruise }}<\alpha_{\max } \\
& S . M \cdot_{\min }<S . M .<S . M ._{\max }
\end{aligned}
$$

Three different optimization algorithms have been used to solve the problem: Complex ${ }^{13-15}$, Fmincon and a Genetic Algorithm. Results are presented in Section V.

\section{E. Penalty Functions}

Some of the problem constraints have been implemented as penalties added to the objective function, so to lead the configuration of the aircraft towards the feasible regions of the design space. The penalty value increases rapidly as soon as the given limit value is passed.

\section{Thickness-To-Chord Ratio Penalty $\left(P_{T C}\right)$}

The first penalty function is aimed at ensuring that the considered configuration allows the engine and the payload bay to be fit inside the aircraft. The actual thickness at the centre line is calculated and compared to the height of the payload bay summed to the engine diameter. The penalty is then calculated as following:

$$
\begin{cases}P_{T C}=K \cdot e^{D_{\text {eng }}+H_{P B}-t_{R} \cdot C_{R}} & \text { if } D_{\text {eng }}+H_{P B}>t_{R} \cdot C_{R} \\ P_{T C}=0 & \text { if } D_{\text {eng }}+H_{P B} \leq t_{R} \cdot C_{R}\end{cases}
$$

where $D_{\text {eng }}$ is the engine diameter, $H_{P B}$ is the height of the payload bay.

2. Angle Of Attack Penalty $\left(P_{\alpha}\right)$

In order to reduce drag and radar cross section, the angle of attack in cruise condition is preferred to be positive and not larger than few degrees. Therefore a penalty function has been implemented as following:

$$
\begin{cases}P_{\alpha}=K_{1, \alpha} e^{\beta_{1}\left(\alpha_{\text {cruise }}-\alpha_{\max }\right)} & \text { if } \alpha_{\text {cruise }}>\alpha_{\max } \\ P_{\alpha}=K_{2, \alpha} e^{\beta_{2}\left(\alpha_{\min }-\alpha_{\text {cruise }}\right)} & \text { if } \alpha_{\text {cruise }}<\alpha_{\min }=0 \\ P_{\alpha}=0 & \text { if } \alpha_{\min }<\alpha_{\text {cruise }}<\alpha_{\max }\end{cases}
$$

where $K_{1, \alpha}, K_{2, \alpha}, \beta_{1}$ and $\beta_{2}$ are constants used to give the wanted weight to the terms in the equations. 


\section{Pitching Moment Penalty $\left(P_{C m}\right)$}

One basic condition the aircraft should fulfil in order to be controllable is to have a negative slope of the pitching moment coefficient as function of the angle of attack, i.e. $C_{m, \alpha}<0$. A function similar to the ones just described has then been introduced to penalize the layouts that do not satisfy this requirement. The function is as following:

$$
\begin{cases}P_{C m}=K_{C m} e^{C_{m, \alpha}} & \text { if } C_{m, \alpha} \geq 0 \\ P_{C m}=0 & \text { if } C_{m, \alpha}<0\end{cases}
$$

As in the previous cases $K_{C m}$ is constant that allows trimming the effect of the penalty function.

\section{Fuel Penalty $\left(P_{\text {fuel }}\right)$}

The goal of the optimization is to define an aircraft layout that is tailored for the specified mission. This means also that the amount of fuel that can be loaded equals the needed quantity. The maximum available fuel tanks volume is estimated considering the total internal volume between the payload bay, the outer wing inner rib, the front and rear wing spars (see Fig. 4). The actual volume that can be used is then reduced taking into account, for simplicity, that the fuel mass should be equally distributed around the centre of gravity (CG). Since the wing becomes thinner as we move towards the trailing edge, the part of the tank in front of CG appears in Fig. 4 smaller than the one after CG.

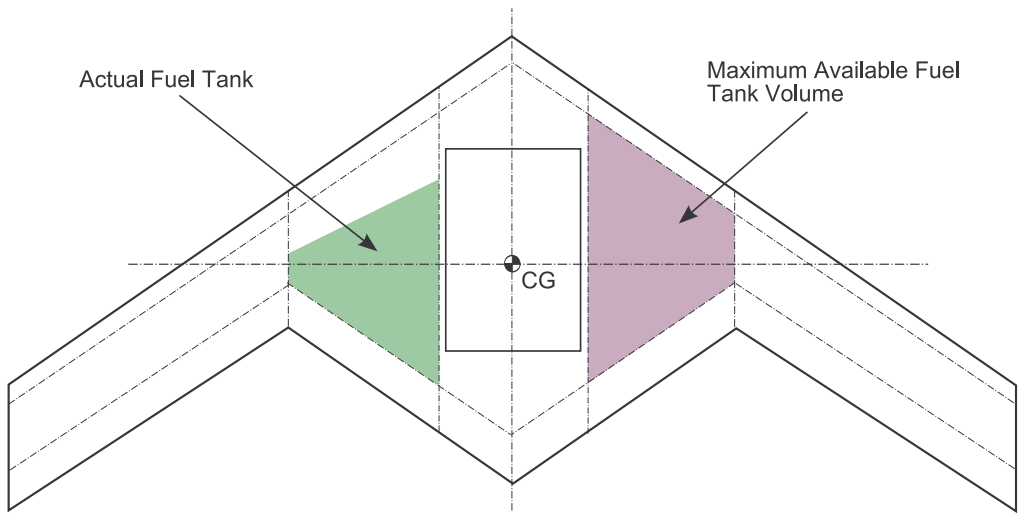

Figure 4. Fuel tank placement

The penalty function has been implemented as follows:

$$
\begin{cases}P_{\text {fuel }}=K_{1, \text { fuel }} \cdot\left(-1+e^{\frac{W_{f, r e q}-W_{f, t}}{W_{f, r e q}}}\right) & \text { if } W_{f, t}<W_{f, r e q} \\ P_{\text {fuel }}=K_{2, \text { fuel }} \cdot\left(-1+e^{\frac{W_{f, t}-W_{f, r e q}}{W_{f, r e q}}}\right) & \text { if } W_{f, t} \geq W_{f, \text { req }}\end{cases}
$$

where $K_{1, f u e l}$ and $K_{2, f u e l}$ are two constants while $W_{f, r e q}$ represents the fuel required to be able to fly the mission and $W_{f, t}$ the maximum fuel quantity that can be loaded into the tanks.

5. Stability Penalty $\left(P_{S}\right)$

The last penalty function implemented takes into account the static margin of the aircraft. For an aircraft of this kind it is not desirable to have a too large static margin, because it would require large trim-deflections of the control surfaces. The aircraft could then result hardly controllable since the surfaces may not be able to be deflected enough before they reach the end position. Therefore it is usual to strive towards small static margins and then relay on the control system. The function used is once again similar to the previous ones:

$$
\begin{cases}P_{S}=K_{1, S} \cdot e^{|S . M .|} & \text { if } S . M .<0 \\ P_{S}=K_{2, S} \cdot e^{|S . M .-S . M .|} \mid & \text { if } S . M . \geq 0\end{cases}
$$

where $K_{l, S}$ and $K_{2, S}$ are two constants and S.M. ${ }^{*}$ is the desired static margin value. 


\section{Test Case: J-UCAS}

The conceptual design of an Unmanned Combat Air Vehicle has been chosen as test case for the presented design optimization tool.

\section{A. Requirements}

The aircraft to be designed have to respond to similar requirements as for the Joint Unmanned Combat Air System (J-UCAS) program. Briefly, this means that the airplane should be autonomous and capable of combat missions of varying nature, such as suppression of enemy defences, precision strike and surveillance and reconnaissance. The main performances required from the aircraft are listed below:

- Payload: $2000 \mathrm{~kg}$

- Combat Radius: $1300 \mathrm{~nm}$

- Persistence: $1000 \mathrm{mn}$ with 2 hrs loiter

- Cruise Speed: $0.8 \mathrm{M}$

- Cruise Altitude: $35000 \mathrm{ft}$

The mission profile that has been taken into consideration is shown in Fig. 5. Slightly different missions can be obtained varying the cruise distances and the loiter endurance, but always within the above specified criteria. Cruise 1 and Cruise 2 are considered to be covering

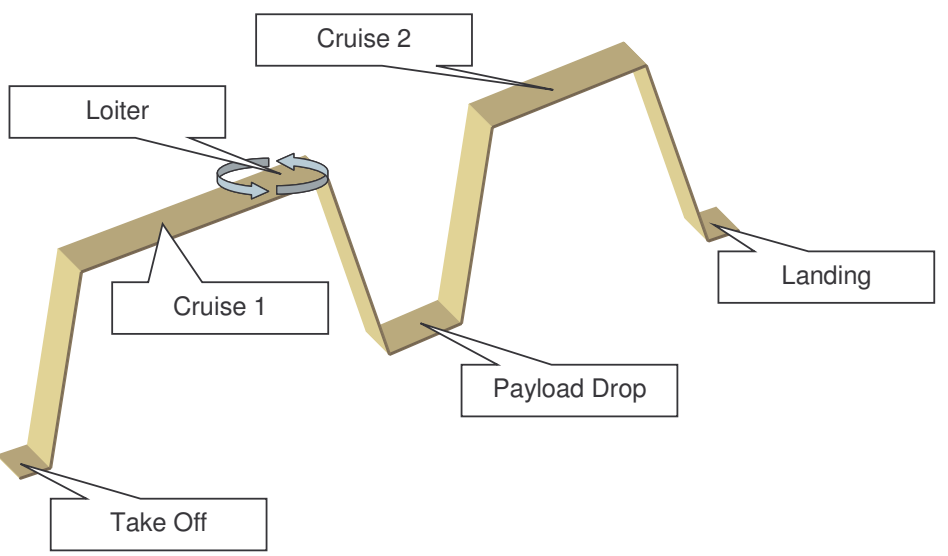

Figure 5. The mission profile the same distance and to be flown at the same height $(35000 \mathrm{ft})$, the only difference being that the payload may only be transported along Cruise 1 .

\section{B. Parametrization}

The outer shape of the aircraft has been linked to the seven parameters in the following list and as illustrated in Fig. 6:

1. Root chord thickness, $t_{R}$

2. Tip chord thickness, $t_{T}$

3. Leading edge sweep, $\Lambda_{L E}$

4. Root chord length, $C_{R}$

5. Tip chord length, $C_{T}$

6. Wing span, $B$

7. Outer wing twist angle, $\rho$

In the centre of the fuselage there shall be space for a payload bay, the dimensions of which are decided by the largest payload the aircraft should be able to carry.

This parametrization is similar to that adopted by Jouannet et al. ${ }^{11}$ In this way the aircraft is described in the same way within the whole framework, granting an easier sharing of information between

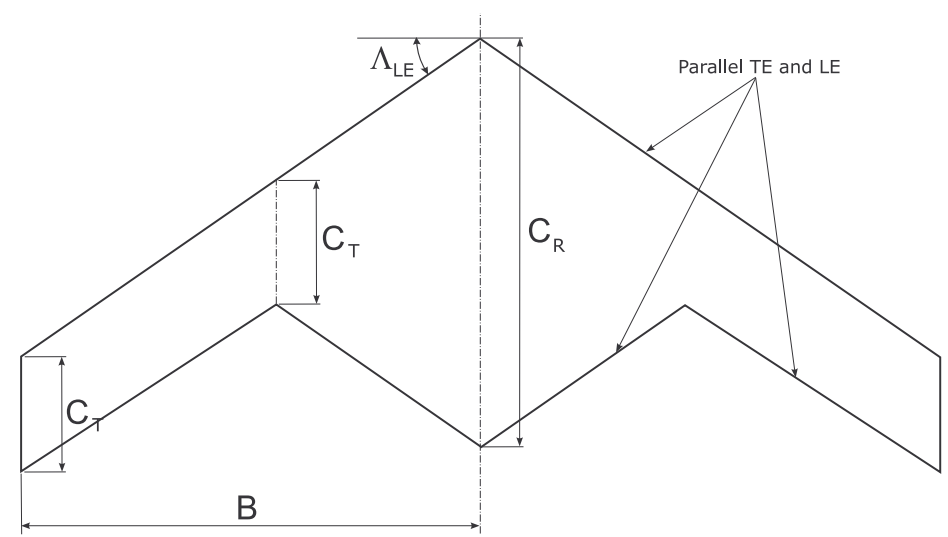

Figure 6. Design parameters defining the outer geometry the modules. The airfoil used all over the airplane is the NACA 4-Digits, but it could be replaced with any other type of airfoil. It is probably not very realistic to use this airfoil only, but having one constant profile type allows reducing significantly the number of parameters, and the modelling complexity. This type of airfoil is defined using 
three parameters: the maximum camber, the position of the maximum camber, and maximum thickness. In the test case only the thickness has been varied, keeping the camber values constant.

\section{Results}

As anticipated in Section III three different optimization algorithms have been tested. The optimizations have been carried out on a personal computer with one single processor AMD Athlon ${ }^{\mathrm{TM}}$ XP 2700+ and 1 GB of RAM. In the following sections the results obtained will be presented. The cycle time for the optimizations was between two and three seconds on the computer used and was mainly depending on the number of nodes used to approximate the airplane's geometry, as described in Section III. A good compromise between accuracy and computing time was found in 616 nodes and 460 panels.

\section{A. Fmincon}

Fmincon is an optimization algorithm that can be found in MatLab's Optimization Toolbox. Quoting the documentation accompanying the algorithm itself, it is a constrained nonlinear optimizer that uses the second order gradient method Sequential Quadratic Programming to find a constrained minimum of a scalar function of several variables starting at an initial estimate.

The use of this algorithm has been unsuccessful for this application. Even though the time required for completing one optimization is usually relatively short, Fmincon seems always to reach to a local optimum of the objective function that corresponds to aircraft layouts far worse than the ones obtained with the other algorithms. Moreover the result is strongly dependent of the starting configuration entered. It has been tried to use configurations (i.e. sets of parameters) only slightly different from one of the solutions obtained with Complex or GA optimizations, hoping that in those cases Fmincon would move towards these solutions, but again without success. Apparently the chosen objective function has many local optimum points that can not be considered as possible absolute optimum and the gradient method locks on them.

\section{B. Complex}

This is a non-gradient optimization algorithm developed from the Simplex method, the main difference being that it uses more points during the search process $^{13}$. Detailed description of the Complex algorithm can be found in Ref. 13, 14 and 15 .

The Complex algorithm has given much better results than Fmincon, but at the cost of longer computing time that during the several tests was registered between 2636 and 5068 seconds, depending on the number of iterations and function evaluations required (see Table 1). The Complex algorithm can be trimmed also tuning on the tolerance for function and parameters convergence, as well as the value of the so called "forgetting factor" and "randomization factor" that in the presented test case have not been modified from the suggested default value 0.3. On the other hand different settings for the tolerance for parameters and function convergence ware tried. Considering that the presented framework is thought to be used during conceptual design phases, where different designs are only roughly described it was felt that it did not make much sense tuning in the design parameter values by changing the third or fourth decimal. The requirement for the objective function convergence was instead much more stringent, since gradients were very small (Fig. 7). The figure shows

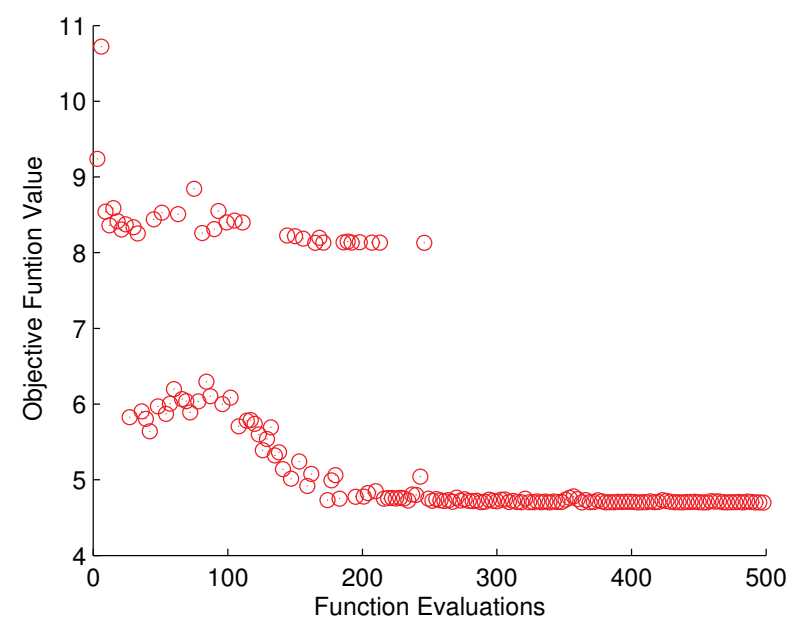

Figure 7. Evolution of the objective function value. The y-axis has a logarithmic scale for enhancing the differences in function values. 
that already after 300 iterations the Complex has reached close to the final configuration. The extension of the flat part of the curve depends on how the convergence requirements have been set. It was observed that even small changes in the objective function value had remarkable effects on the maximum take-off weight. Thus the Complex was set to continue until the relative change in function value was less then $10^{-4}$. The convergence requirement for the parameters' values was set instead to $5 \cdot 10^{-3}$.

Although performing much better than Fmincon, the Complex algorithm did not always reach to what is believed to be a possible absolute optimum. Among all the tests performed, the best parameters set hit rate was $17 \%$. Figure 8 shows the suggested configuration while its parameter set is reported in Table 2; the calculated maximum take-off weight is $18,716 \mathrm{~kg}$. It has anyway to be noted that, among all the solutions found by the Complex algorithm, the difference between the best and the worst configuration measured in maximum take-off weight is of less than 400 $\mathrm{kg}$, from 18.7 tons to 19.1 tons.

\begin{tabular}{|l|c|c|c|c|c|c|c|}
\hline Parameter & $\boldsymbol{t}_{\boldsymbol{R}}$ & $\boldsymbol{t}_{\boldsymbol{T}}$ & $\boldsymbol{\Lambda}_{\boldsymbol{L E}}$ & $\boldsymbol{C}_{\boldsymbol{R}}$ & $\boldsymbol{C}_{\boldsymbol{T}}$ & $\boldsymbol{B}$ & $\boldsymbol{\rho}$ \\
\hline Value & $20 \%$ & $8.13 \%$ & $44.2^{\mathrm{o}}$ & $13.49 \mathrm{~m}$ & $4.17 \mathrm{~m}$ & $5.99 \mathrm{~m}$ & $2.56^{\circ}$ \\
\hline
\end{tabular}

Table 2. Design parameters of the suggested configuration using the Complex algorithm.
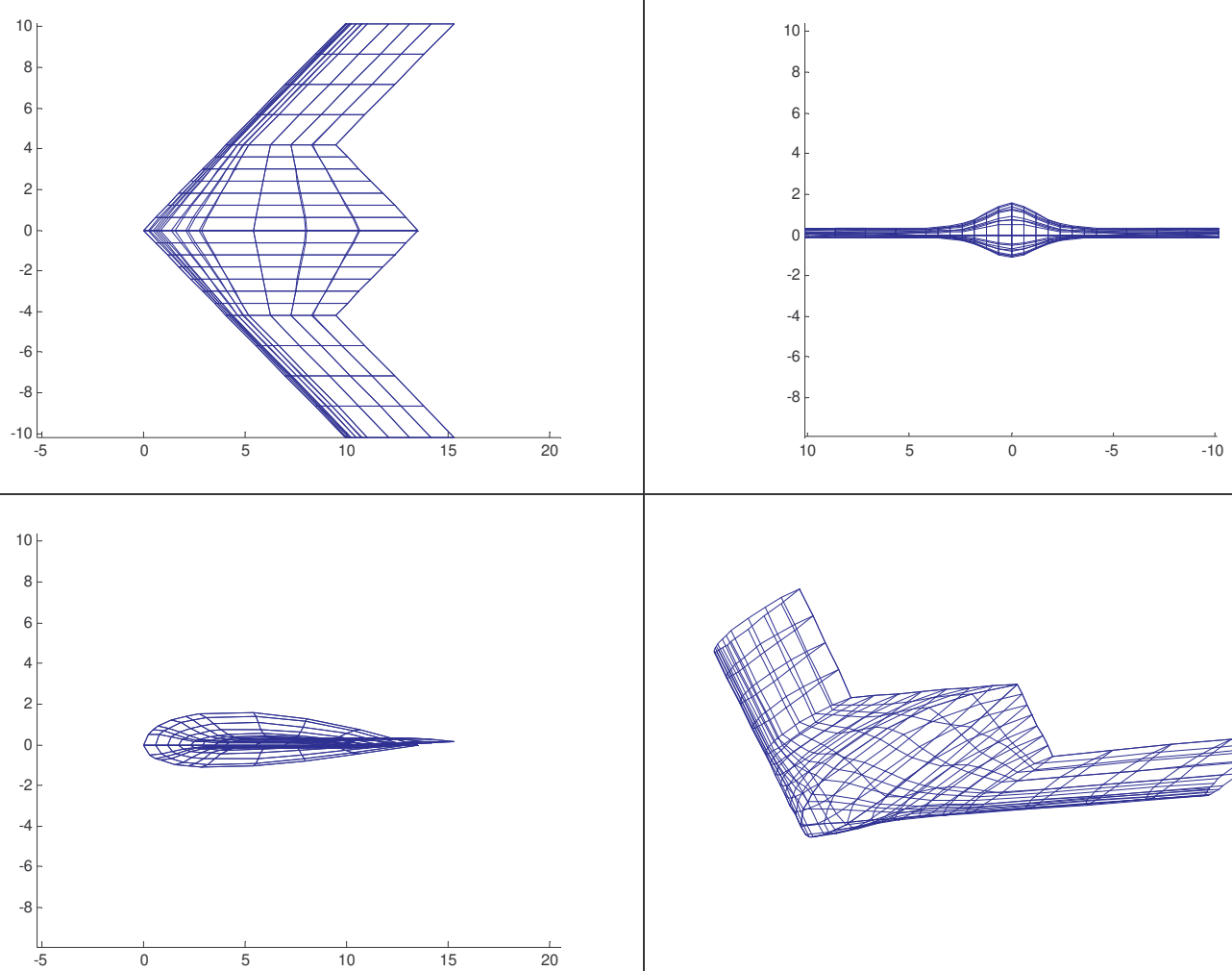

Figure 8. Suggested best configuration using the Complex algorithm.

\section{Genetic Algorithm}

The last optimization method tested is a genetic algorithm (GA). The basic idea of GAs is the mechanics of natural selection. Each optimization parameter, $\left(x_{n}\right)$, is coded into a gene as for example a real number or string of bits. The corresponding genes for all parameters, $x_{1}, \ldots x_{n}$, form a chromosome, which describes each individual. A chromosome could be an array of real numbers, a binary string, a list of components in a database, all depending on the specific problem. Each individual represents a possible solution, and a set of individuals form a population. In a population, the fittest are selected for mating. Mating is performed by combining genes from different parents to produce a child, called a crossover. There is also a possibility that a mutation might occur which change the value of a gene randomly. Finally the children are inserted into the population to form a new generation. The procedure starts 
over again, thus representing an artificial Darwinian environment. The optimization continues until the population has converged or the maximum number of generations has been reached. The implemented GA uses real encoding, roulette wheel selection, extended intermediate recombination, mutation according to the breeder GA, linear fitness scaling and an elitist replacement strategy where the $G_{G a p}$ percentage of the weakest individuals is replaced in each generation. The present GA used a population size of 40 individuals and was run for 100 generations with a crossover probability of 0.6 and a generation gap of 0.8 .

Compared to the other algorithms it registered the longest computing time due to the larger number of iterations and objective function evaluations required. The total computing time was around 2.5 hours that is in line with data from Table 1. On the other hand, using this genetic algorithm the hit rate rose to $75 \%$. One criterion for comparing the Complex and GA could be to consider following performance index definition?

$$
f_{\text {obj }, o p t}=-\frac{\log _{2}\left(1-P_{o p t}\right)}{k_{m}}
$$

where $P_{\text {opt }}$ is the probability of finding the optmum and $k_{m}$ is the number of iterations required. According to this definition the performance index of the GA is only three times better than the Complex algorithm. The difference is in fact not as big as it might seem at first.

Figure 9 shows the best configuration obtained and Table 3 its design parameters. The calculated maximum takeoff weight for this aircraft is $18,656 \mathrm{~kg}$, which is almost exactly the same value obtained with the Complex algorithm. It should be remarked that all the configurations obtained with the genetic algorithm that seems to be absolute optima do not have the same calculated take-off weight: among all the tests carried out there is a variation

\begin{tabular}{|l|c|c|c|c|c|c|c|}
\hline Parameter & $\boldsymbol{t}_{\boldsymbol{R}}$ & $\boldsymbol{t}_{\boldsymbol{T}}$ & $\boldsymbol{\Lambda}_{\boldsymbol{L} \boldsymbol{E}}$ & $\boldsymbol{C}_{\boldsymbol{R}}$ & $\boldsymbol{C}_{\boldsymbol{T}}$ & $\boldsymbol{B}$ & $\boldsymbol{\rho}$ \\
\hline Value & $20 \%$ & $8.15 \%$ & $44.11^{\circ}$ & $13.50 \mathrm{~m}$ & $4.21 \mathrm{~m}$ & $6.00 \mathrm{~m}$ & $2.20^{\circ}$ \\
\hline
\end{tabular}

Table 3. Design parameters of the suggested best configuration using the genetic algorithm.
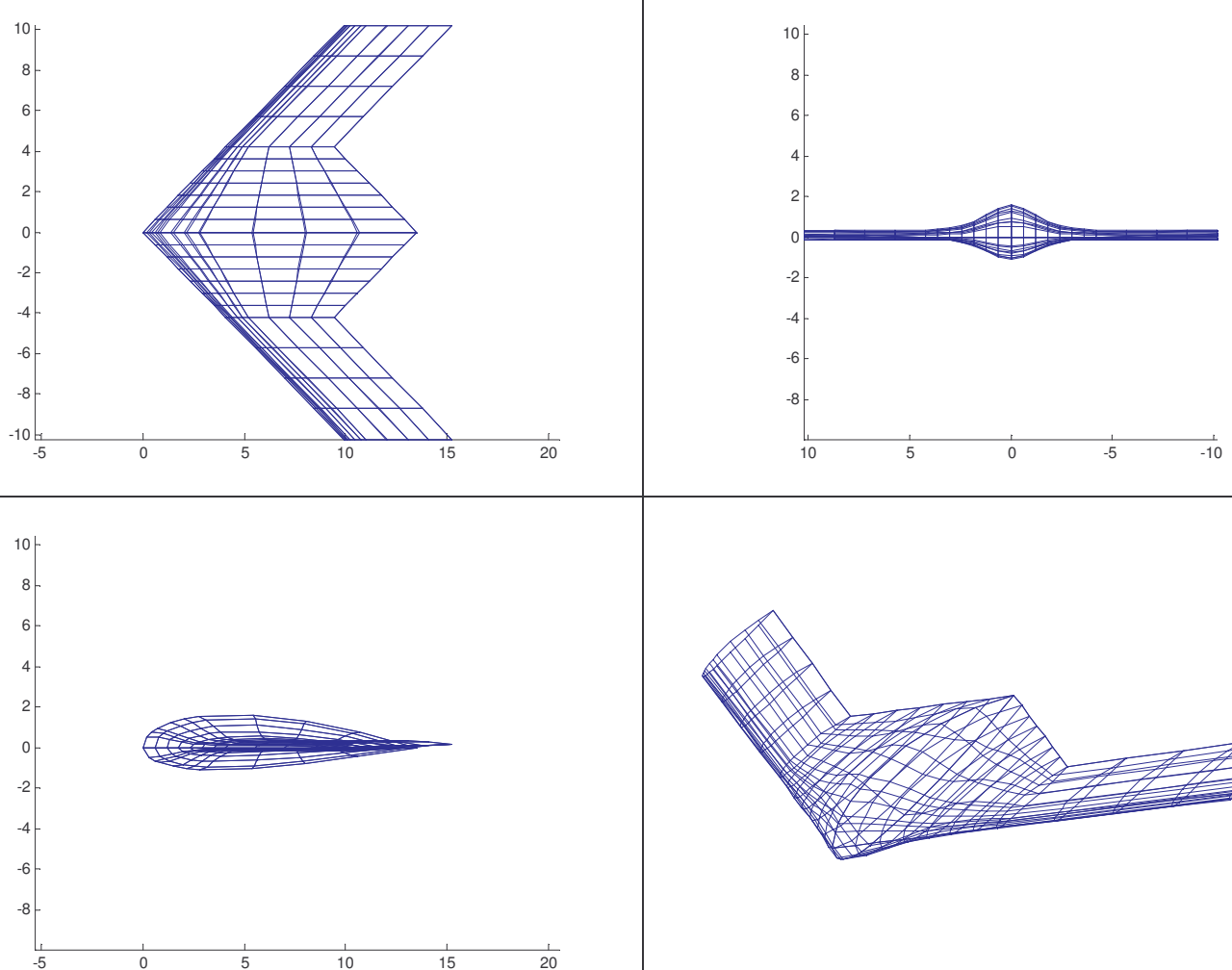

Figure 9. Suggested best configuration using the genetic algorithm. 
of $0.95 \%$. The objective function value diagram in Fig. 10 shows that there can be a big difference in the way the algorithm finds its way to the optimum. In both the cases the suggested configuration is exactly the same.
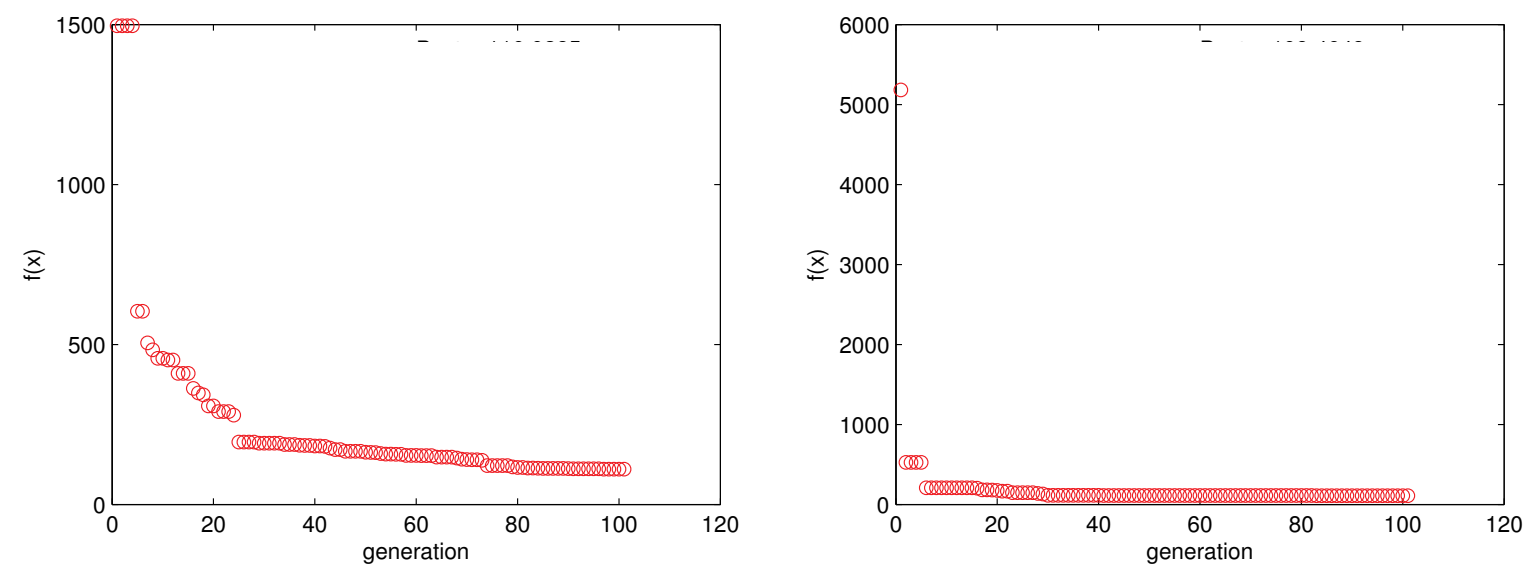

Figure 10. Evolution of the objective function value for two different optimizations using the genetic algorithm.

\section{Conclusion and Future Developments}

A novel framework for aircraft conceptual design is being developed at Linköping University. Specifically this work illustrates how the aerodynamic analysis module is structured and how it can be linked to an optimization algorithm for analyzing different aircraft concepts in order to identify the one or ones that appear to be best suited to fulfill the specified mission. Three different optimization algorithms have been tested and their performances compared with each other, showing that gradient methods are not able to solve the present problem and that a nongradient method algorithm is mandatory, in this case the Complex and a genetic algorithm. Results show that, even though the Complex algorithm is about two times faster on the single optimization, the genetic algorithm ensures a by far better hit rate (75\% against $17 \%$ ), which makes the genetic algorithm to be preferred.

To validate the presented framework module the design of an unmanned aerial vehicle was considered. Its mission profile and requirements were very similar to the J-UCAS program. The configuration suggested by the optimization analysis is in fact very similar in size and weight to the Boeing X-45C and Northrop Grumman X-47B. The differences in shape between the presented layouts and the above mentioned aircrafts is due to the constraints specified in the parametrization of the model.

The development of the framework is in full progress. A fully parametric three dimensional CAD model comprising structure and sub-systems is being created with CATIA V5 and it will be tried to use it for generating the input data for the aerodynamic analysis. Work is also carried out on improving the interface between user and framework. Finally other modules are being integrated in the framework and new ones are being developed.

\section{References}

${ }^{1}$ Röhl, P.J., Mavris, D.M., Schrage, D.P., “Combined Aerodynamic and Structural Optimization of a High-Speed Civil Transport Wing", $6^{\text {th }}$ AIAA Structures, Dynamics, and Materials Conference, New Orleans, LA, 1995.

${ }^{2}$ Morris, A., Arendsen, P., LaRocca, G., Laban, M., Voss, R., Hönlinger, H., "MOB - A European Project on Multidisciplinary Design Optimization", $24^{\text {th }}$ International Congress Of The Aeronautical Sciences, Yokohama, Japan, 2004

${ }^{3}$ Lovell, D.A., Crawford, C.A., Restrick, K.E., "Recent Advances In Air-Vehicle Design Synthesis And Optimisation", $24^{\text {th }}$ International Congress Of The Aeronautical Sciences, Yokohama, Japan, 2004.

${ }^{4}$ Dovi, A.R., Wrenn, G.A., Barthelemy, J.-F.M., Coen, P.G., Hall, L.E., "Multidisciplinary Design Integration Methodology for a Supersonic Transport Aircraft”, Journal of Aircraft, Vol. 32, No. 2, 1995, pp. 290-296.

${ }^{5}$ Goraj, Z., Frydrychewicz, A., Hermetz, J., Le Tallec, C., "Hale UAV Platform Optimised for a Specialized 20-km Altitude Patrol Mission", $24^{\text {th }}$ International Congress Of The Aeronautical Sciences, Yokohama, Japan, 2004.

${ }^{6}$ Sevant, N.E., Bloor, M.I.G., Wilson, M.J., "Cost Effective Multipoint Design of a Blended High-Speed Civil Transport", Journal of Aircraft, Vol. 36, No. 4, 1999, pp. 642-650.

${ }^{7}$ Johansson, B., Jouannet, C., Krus, P., "Distributed Aircraft Analysis Using Web Service Technology", World Aviation Congress ${ }_{\circledast}$ \& Exposition, Montreal, Canada, 2003.

${ }^{8}$ Raymer, D.P., “Aircraft Design: A Conceptual Approach”. Second Edition, AIAA Educational Series, 1992. 
${ }^{9}$ Erikson, L.L., "Panel Methods - An Introduction”, NASA Technical Paper 2995, 1990

${ }^{10}$ Towne, M.C., Strande, S.M., Erikson, L.L., Kroo, J.M., Enomoto, F.Y., Carmichael, R.L., McPherson, K.F., "PAN AIR Modelling Studies”, AIAA Applied Aerodynamics Conference, Danvers, Massachusetts, 1983.

${ }^{11}$ Jouannet, C., Silva, S.E.R., Krus, P., "Use Of CAD For Weight Estimation In Aircraft Conceptual Design", $24^{\text {th }}$ International Congress Of The Aeronautical Sciences, Yokohama, Japan, 2004.

${ }^{12}$ Jenkinson, L.R., Simpkin, P., Rhodes, D., "Civil Jet Aircraft Design”, Butterworth-Heinemann Publications, 1999.

${ }^{13}$ Andersson, J., "Design Optimization”, Linköping University, Linköping, Sweden, 2005.

${ }^{14}$ Krus, P., Andersson, J., “An Information Theoretical Perspective On Design Optimization”, Design Engineering Technical Conference, Salt Lake City, Utah, USA, 2004.

${ }^{15}$ Krus, P., Andersson, J., "Optimizing Optimization for Design Optimization", ASME Design Automation Conference, Chicago, Illinois, USA, 2003.

${ }^{16}$ Ardema, M.D, Chambers, M.C., Patron, A.P., Hahn, A.S., Miura, H., Moore, M.D., “Analytical Fuselage And Wing Weight Estimation Of Transport Aircraft”. NASA TM 110392, May 1996.

${ }^{17}$ Katz, J., Plotkin, A., "Low Speed Aerodynamics", Second Edition, Cambridge University Press, 2001. 